\title{
Comparison of Neuromuscular Injuries to the Surgeon during Hand-Assisted and Standard Laparoscopic Urologic Surgery
}

WILLIAM K. JOHNSTON, III, M.D.,* BRENT K. HOLLENBECK, M.D., and J. STUART WOLF, JR., M.D.

\begin{abstract}
Background and Purpose: Hand-assisted procedures have assumed a greater role in the practice of many laparoscopists. We surveyed major laparoscopy program directors to compare the incidence and location of neuromuscular injury to the surgeon during hand-assisted laparoscopic (HAL) and standard laparoscopic (SL) surgery.

Materials and Methods: A questionnaire on neuromuscular injuries was e-mailed to 42 laparoscopic program directors. Respondents were instructed to report only injuries or pain associated with laparoscopic surgery when they were the primary responsible surgeon and not during open or endoscopic procedures.

Results: Surveys were returned from 23 attending laparoscopic surgeons and 2 laparoscopic fellows. Surgeons reported an average of 3.9 HAL and 6.3 SL cases per month as the primary surgeon. The HAL was completed with the GelPort, LapDisk, Omniport, or a combination of devices $55 \%, 22 \%, 5 \%$, and $14 \%$, respectively, of the time. Comparing HAL with SL, there was significantly more hand/wrist, forearm, and shoulder pain/injuries associated with HAL $(P<0.004)$. There was significantly more neck pain associated with SL than HAL $(P<\mathbf{0 . 0 0 3})$, but no significant difference in lower-back pain $(P=0.40)$. Comparing the two most commonly used hand-assist devices (GelPort and LapDisk), the LapDisk demonstrated significantly more hand/wrist pain or injury $(P=0.001)$.

Conclusion: Hand-assisted laparoscopy is associated with more frequent neuromuscular strain to the upper extremity than SL, but SL surgeons experience more neck pain or injury. Surgeon discomfort is also dependent on the type of hand-assist device. The long-term consequences of physical strain on the laparoscopic surgeon are unknown currently, but measures to minimize neuromuscular strain should be considered.
\end{abstract}

\section{INTRODUCTION}

$\mathbf{S}$ TANDARD LAPAROSCOPY (SL) can be technically difficult because of demanding pathologies, large tumors, or severely scarred and inflamed tissue. Furthermore, for lessexperienced laparoscopic surgeons, operative times may be lengthy because of limitations imposed by instrument position and length and the lack of tactile feedback. Accordingly, handassisted laparoscopy (HAL) was developed to improve the speed and safety of laparoscopic procedures by less-experienced surgeons or when there is demanding pathology, or intact specimen retrieval is desired. ${ }^{1,2}$

Advancements in technology and medical devices have helped foster the development of minimally invasive surgery. Yet with advancements in minimally invasive surgery, unique ergonomic challenges have surfaced because of the atypical positions and limited degrees of freedom to which surgeons are exposed during HAL and SL. In 2000, we reported a multi-institutional study that surveyed the physical strain on the surgeon during laparoscopy. ${ }^{3}$ With the continuing expansion of HAL and our own anecdotal experience that HAL was in many ways more physically stressful than SL, we hypothesized that HAL made the surgeon more susceptible to pain or discomfort than SL. To assess this question, we surveyed directors of major laparoscopy programs to compare the incidence and location of neuromuscular injuries to the surgeon during HAL and SL.

\section{MATERIALS AND METHODS}

A questionnaire relating to neuromuscular injuries was emailed to 42 laparoscopy program directors (Fig. 1). Surgeons 


\begin{tabular}{|c|c|c|c|c|c|c|}
\hline \multicolumn{7}{|c|}{ I. HAND-ASSISTED LAPAROSCOPY: } \\
\hline & Usually & Frequently & \multicolumn{2}{|c|}{ Occasionally } & Rarely & Never \\
\hline \multicolumn{7}{|l|}{ Neck/Upper Back pain or injury } \\
\hline \multicolumn{7}{|l|}{ Lower back pain or injury } \\
\hline \multicolumn{7}{|l|}{ Hand/wrist pain or injury } \\
\hline \multicolumn{7}{|l|}{ Forearm/elbow pain or injury } \\
\hline \multicolumn{7}{|l|}{ Shoulder pain or injury } \\
\hline $\begin{array}{l}\text { What type of hand-port do } \\
\text { you routinely use? }\end{array}$ & Gelport & Lap Disc & $\begin{array}{l}\text { Omni } \\
\text { port }\end{array}$ & \multicolumn{2}{|c|}{ Other } & \\
\hline $\begin{array}{l}\text { How many HAL cases do } \\
\text { you average per month } \\
\text { as responsible surgeon? }\end{array}$ & \multicolumn{3}{|c|}{$\begin{array}{l}\text { Which hand do you } \\
\text { use for L. Kidneys? }\end{array}$} & \multicolumn{2}{|c|}{$\begin{array}{l}\text { Which hand do } \\
\text { you use for R. } \\
\text { Kidneys? }\end{array}$} & \\
\hline \\
\hline \multicolumn{7}{|l|}{ II. STANDARD LAPAROSCOPY } \\
\hline \multirow{2}{*}{\multicolumn{7}{|c|}{$\begin{array}{l}\text { Neck/Upper Back pain or injury } \\
\text { Lower back pain or in jury }\end{array}$}} \\
\hline & & & & & & \\
\hline \multicolumn{7}{|l|}{ Hand/wrist pain or injury } \\
\hline \multicolumn{7}{|l|}{ Forearm/elbow pain or injury } \\
\hline \multicolumn{7}{|l|}{ Shoulder pain or injury } \\
\hline $\begin{array}{l}\text { How many SL cases do yo } \\
\text { ave rage per month as resp } \\
\text { surgeon? }\end{array}$ & onsible & & & & & \\
\hline
\end{tabular}

FIG. 1. Survey of neuromuscular injury or pain during laparoscopy.

were instructed to report the number of cases and type of laparoscopic approach (HAL and SL) they averaged per month. They were also asked to report injuries or pain experienced with laparoscopic surgery when they were the primary responsible surgeon (and not those occurring during open or endoscopic procedures). Questions focused on pain at the hand/wrist, forearm, shoulder, neck, and lower back. Respondents were asked to check the box that best described their frequency of pain ("usually," "frequently," "occasionally," "rarely," and "never"). For those surgeons who perform HAL, the type of hand port routinely used was asked about. Statistical analysis was completed with the chi-square test.

\section{RESULTS}

Twenty-five surveys (60\%) from 23 attending laparoscopic surgeons and 2 laparoscopic fellows were returned. Surgeons reported an average of 3.9 HAL and 6.3 SL cases per month as the primary surgeon.

There were significantly more hand/wrist, forearm, and shoulder pain/injuries associated with HAL $(P<0.004)$. For hand/wrist pain, $33 \%$ of HAL $v 8 \%$ of SL led to pain "usually/frequently" (Fig. 2). Forearm and shoulder pain were experienced "usually/frequently" by $25 \%$ and $10 \%$, respectively, during HAL $v 4 \%$ and 0 during SL (Fig. 3). Compared with upper-extremity pain in both HAL and SL, surgeons reported lessfrequent problems with neck and back discomfort. During HAL, $85 \%$ and $65 \%$ of the surgeons $v 60 \%$ and $66 \%$ of the SL sur- geons "rarely" or "never" experience neck and back pain, respectively. Nonetheless, there was significantly more neck pain associated with SL than HAL $(P<0.003)$ but no significant difference in lower-back pain $(P=0.40)$ (Fig. 4).

The HAL was completed with the GelPort, LapDisk, Omniport, or a combination of devices in $55 \%, 22 \%, 5 \%$, and $14 \%$ of the cases, respectively. Comparing the two most commonly used hand-assisted devices (GelPort and LapDisk), the LapDisk demonstrated significantly more hand/wrist pain or injury $(P=$ $0.001)$.

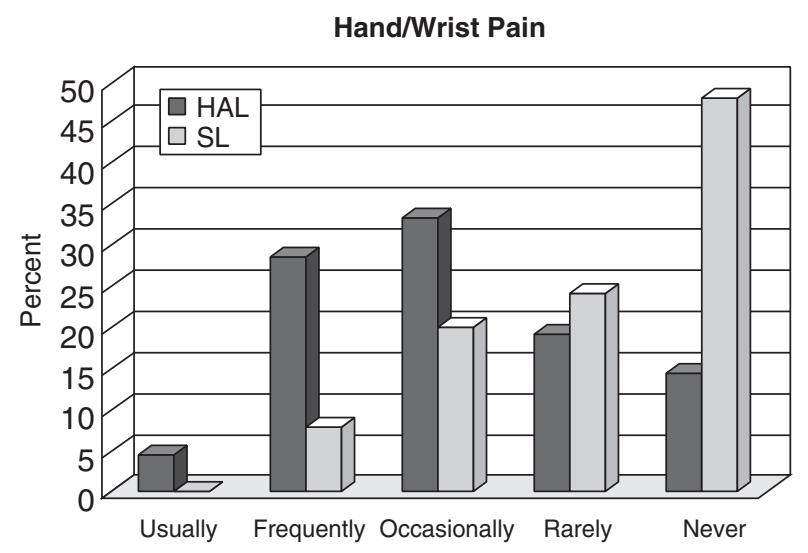

FIG. 2. Neuromuscular injuries to surgeon's hand/wrist during HAL $v$ SL. 
A

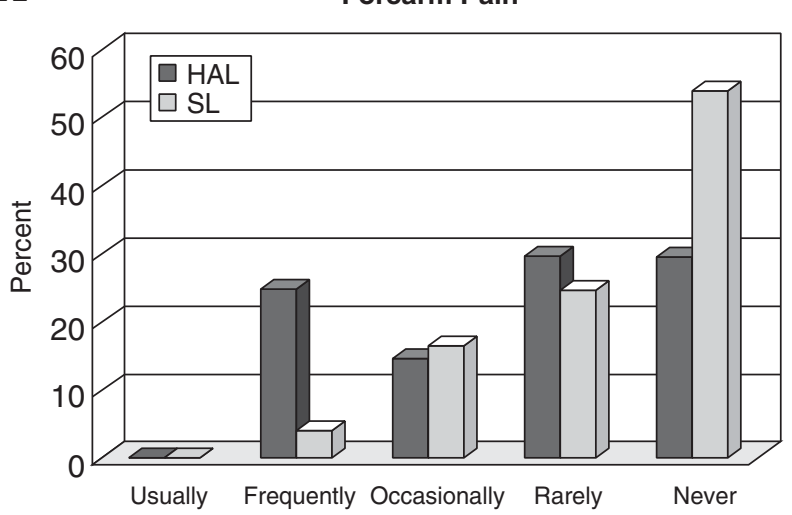

B

Shoulder Pain

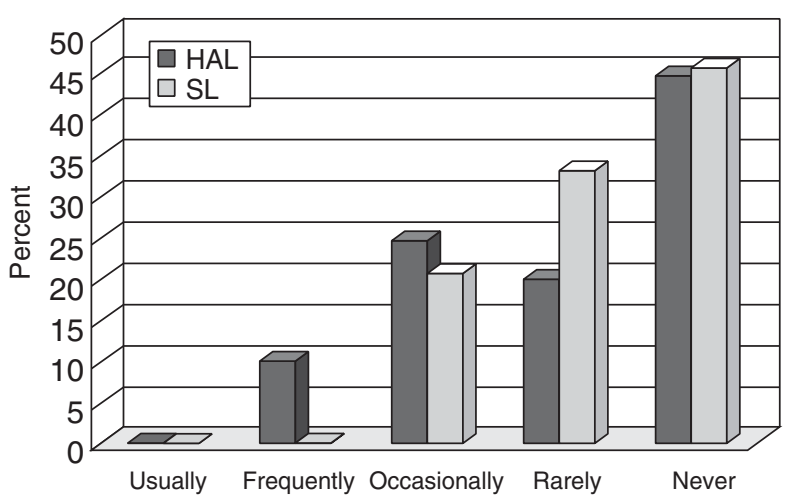

FIG. 3. Neuromuscular injuries to surgeon's forearm (A) and shoulder (B) during HAL $v$ SL.

\section{DISCUSSION}

Laparoscopy has assumed a much greater role in urology today and continues to grow as more urologists are trained in minimally invasive surgery. ${ }^{4}$ While slow in gaining momentum initially for operations such as pelvic lymphadenectomy, enthusiasm began to mount after Clayman and associates ${ }^{5}$ performed the first laparoscopic nephrectomy. This report set off a wave of laparoscopic urology that has at this point encompassed almost every urologic operation. Laparoscopic surgery has changed the environment in which surgeons interact with the patient, and ergonomics, the study of the interaction between humans and their working environment, has become more important as the neuromuscular strain on the laparoscopic surgeon becomes more evident. ${ }^{6}$ For the surgeon trying to balance costs and still maximize quality and functional design in laparoscopic equipment, ergonomic design usually finds itself low on the priority list in choosing surgical instruments. ${ }^{7}$ Furthermore, concurrent improvements in operating-room design and video-monitor positioning have been slow. As a result, technology has outpaced ergonomic upkeep, and surgeons are exposed to muscle fatigue and risk chronic injury during laparoscopy.

Fatigue and muscle pain results from repetitive movements or isometrics beyond that normally exerted on a muscle group. In open surgery, surgeons frequently complain of neck and back pain, usually related to the need to obtain better vision and exposure by assuming less ergonomically appropriate positions. Laparoscopy eliminates the need to reposition the body or neck to gain a better view during surgery. Nonetheless, surgeons expose themselves to awkward motions and static positioning owing to the awkward instrumentation, which can lead to muscular strain during surgery. ${ }^{8}$

The introduction of HAL has facilitated urologic surgeons' entry into laparoscopic surgery, allowing tactile feedback, finger dissection, manual control of bleeding, and shorter operating times. ${ }^{9,10}$ However, HAL has also introduced new ergonomic challenges beyond those of SL, as it restricts working space and inhibits free, synchronized movement of the hand, wrist, forearm, and shoulder. A HAL operation may also necessitate a more lordotic posture, which may contribute to back and neck pain. ${ }^{10}$

In our study, there was significantly more neck pain associated with SL than with HAL but no significant difference in back pain. There are multiple reasons SL may contribute to more neck pain than HAL. First, SL cases typically are longer than HAL,${ }^{10}$ thus exposing the neck to more strain. Also, surgeon monitors are not always positioned properly, with the monitor placed in the direct line of vision. Consequently, surgeons may maintain their necks in a flexed or turned position for extended periods of time. During HAL, surgeons typically alter their position more frequently and are less likely to subject their necks to prolonged static positions that result in mus-

A

Neck Pain

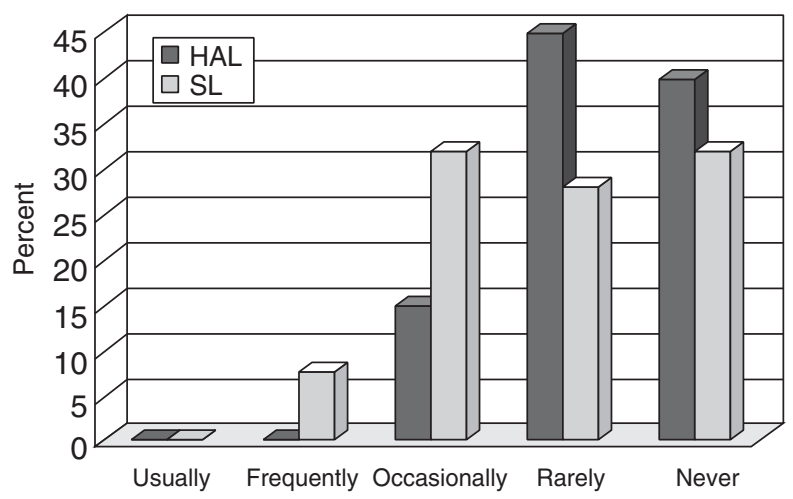

B

Lower Back Pain

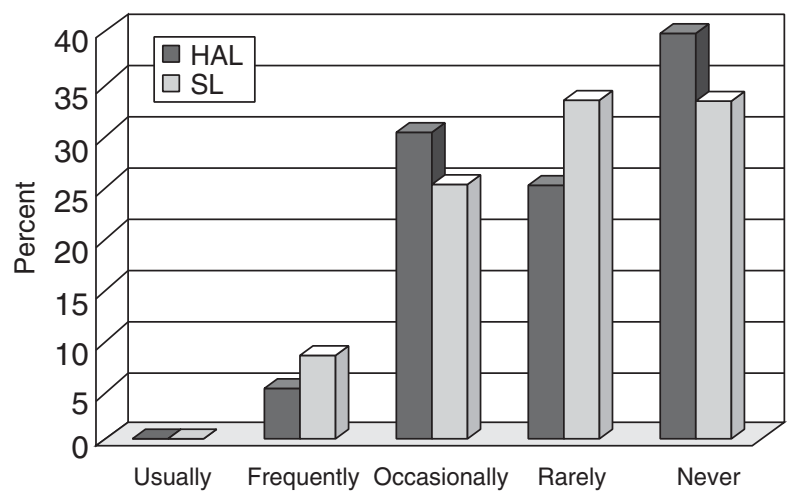

FIG. 4. Neuromuscular injuries to surgeon's neck (A) and back (B) during HAL $v$ SL. 
cle fatigue and injury. ${ }^{11}$ Surprisingly, HAL did not cause significantly more back pain than SL. Perhaps shorter case times or reduced torso movement played a role in this finding, although we did not request average surgical times for HAL and SL in our survey. Berguer and associates ${ }^{12}$ found less back pain but more neck pain when comparing laparoscopic with open surgery. Those investigators attributed the differences to a more upright posture but less anteroposterior weight shifting during laparoscopy.

When comparing HAL and SL, wrist/hand, forearm, and shoulder pain were more common complaints with the former. These results most likely reflect the awkward angles assumed by each portion of the upper extremity during HAL. The fact that the hand/wrist were the part of the extremity most commonly experiencing pain during HAL relates to the relatively fixed position of the wrist, while the hand distally and the forearm proximally are paradoxically fulcrumed against one another within the hand port. Furthermore, HAL involves complex finger positions that tax muscles in short periods of time. Overall, these unusual hand and finger positions may lead to spasm and pain necessitating rest and recuperation before continuing with surgery.

There are also problems inherent in each hand-port's design that contribute to the wrist/hand pain. For instane, Monga and associates ${ }^{13}$ found that the GelPort and LapDisk exerted the most forearm compression and the Handport the least compression. The Handport and PneumoSleeve afford the best comfort at the price of being more cumbersome, with their requirement for a separate sleeve. During the study by Monga and associates, the Omniport was not evaluated, but subjectively, we feel strongly that it creates less compression than the GelPort or LapDisk. Comparing the two most commonly used hand-assist devices in our study (GelPort and LapDisk), the LapDisk demonstrated significantly more hand/wrist pain.

Our findings can be compared with those of a study completed by the Society of American Gastrointestinal Endoscopic Surgeons. Of the 149 responding surgeons, 12\% reported pain in the upper extremity and $8 \%$ neck pain. An ergonomic station showed that laparoscopic grasping entailed more peak and total forearm and thumb muscle effort than hemostatic grasping (open technique). ${ }^{14}$ The fact that our study found a higher percentage of upper-extremity pain with both SL and HAL may reflect the length of surgery or port positioning. The neck pain associated with SL was similar to their findings. ${ }^{14}$ In 1999, a survey of surgeon pain associated with urologic laparoscopic surgery found that $67 \%, 11 \%, 17 \%, 28 \%$, and $33 \%$ reported at least occasional pain in the hand or wrist, elbow, shoulder, neck pain, and back, respectively. ${ }^{3}$ These problems were not stratified by laparoscopic approach (SL $v$ HAL). Over the last 5 years since that study was done, gradual improvements have been made in instrument design, operating-room layout, and surgical approach, yet our data suggest that surgeons still experience significant discomfort. These groups are not entirely comparable because of changing variables such as decreased length of certain operations and increased complexity of others such as reconstructive surgery and lower-tract surgery (radical prostatectomy). Our survey did not examine these factors.

As laparoscopic surgery expands to include more complex tasks such as suturing and knot tying, the degree of muscle fatigue and injury would be expected to increase. In fact, Quick and associates ${ }^{15}$ found greater activation of all upper-extrem- ity muscles during complex (cable-tying) than simple (grasping) laparoscopic exercises. Focal muscle fatigue may influence other muscle groups not normally involved in the activity. As fatigue increases, surgeons may find alternative joint and limb positions to compensate for muscle exhaustion. For instance, during HAL, bending at the waist allows the surgeon to use the entire arm for rotation, reducing the work of the forearm but increasing the work of the deltoid. In this position, the surgeon must increase the flexion of the neck to view the screen. Thus, compensation for fatigue in one muscle has been reduced by shifting the work to other areas of the limb and torso, and this ultimately may lead to injury at sites away from the primary muscles being used.

Proper design and fitting of laparoscopic instruments may minimize neuromuscular strain. Surgical-port placement can affect surgeon comfort. Placement of the ports with the proper spacing and positioning in line with the surgeon can reduce muscle stress. Berguer and colleagues ${ }^{16}$ also examined the effects of working angles on surgeon's upper-extremity workload. They found that instrument alignment in the extreme horizontal position increases forearm and shoulder muscle work and, in the extreme vertical position, escalates shoulder fatigue. However, neither position significantly affects the thenar muscles. Nonetheless, in some procedures, ideal port and instrument positions cannot be entirely entertained.

Initial planning of operating rooms should consider ergonomics and should keep pace with the development and implementation of new technology. Berguer et $\mathrm{al}^{17}$ examined the optimal table height for SL and found the least surgeon discomfort and upper arm and shoulder work when the laparoscopic instrument handles were at the level of or $10 \mathrm{~cm}$ below the surgeon's elbow. Similar findings were described by van Veelen and coworkers. ${ }^{18}$ Current limits of operating-room tables may preclude reaching this position, especially in patients with large body habitus or positioned in the flank, Trendelenburg, and reverse Trendelenburg. Building platforms with steps is the most immediate remedy, but this causes its own restriction of surgeon movement, comfort, and safety. Monitor positioning and height affect muscle fatigue, particularly in the neck muscles, ${ }^{19}$ and positioning the monitor closer to the operative field has been reported to improve performance. ${ }^{20}$

Surgeon pain during laparoscopy is most likely multifactorial. Our study was limited in its focus to whether there are differences in neuromuscular pain between HAL and SL. The size of our cohort and the brevity of the questionnaire precluded the ability to examine some of the specific factors that influence pain during laparoscopy beyond the hand port.

\section{CONCLUSION}

Hand-assisted laparoscopy is associated with more neuromuscular strain on the surgeon's upper extremity than SL. However, SL surgeons experience more neck pain. During HAL, the extent of discomfort may depend on type of hand device used. The longterm consequences of physical strain on the laparoscopic surgeon are unknown currently, but with the expanding application of laparoscopy in urology, measures to minimize neuromuscular strain should be considered. The study of ergonomics during laparoscopy demands ongoing vigilance and commitment to improve the 
working conditions of the surgeon and support the bonding of surgeons to their instruments. Ultimately, maximizing these efforts will result in less surgeon pain and fatigue, increased and prolonged productivity, and greater patient safety.

\section{REFERENCES}

1. Hollenbeck BK, Seifman BD, Wolf JS Jr. Clinical skills acquisition for hand-assisted laparoscopic donor nephrectomy. $\underline{\mathrm{J} \text { Urol }}$ 2004;171:35.

2. Kercher KW, Joels CS, Matthews BD, Lincourt AE, Smith TI, Heniford BT. Hand-assisted surgery improves outcomes for laparoscopic nephrectomy. Am Surg 2003;69:1061.

3. Wolf JS Jr, Marcovich R, Gill IS, et al. Survey of neuromuscular injuries to the patient and surgeon during urologic laparoscopic surgery. Urology 2000;55:831.

4. Shay BF, Thomas R, Monga M. Urology practice patterns after residency training in laparoscopy. J Endourol 2002;16:251.

5. Clayman RV, Kavoussi LR, Soper NJ, et al. Laparoscopic nephrectomy: Initial case report. J Urol 1991;146:278.

6. Stone R, McCloy R. Ergonomics in medicine and surgery. BMJ 2004;328:1115.

7. Van Veelen MA, Meijer DW. Ergonomics and design of laparoscopic instruments: Results of a survey among laparoscopic surgeons. J Laparoendosc Adv Surg Tech A 1999;6:481.

8. Nguyen NT, Ho HS, Smith WD, Philipps C, Lewis C, De Vera RM, Berguer R. An ergonomic evaluation of surgeons' axial skeletal and upper extremity movements during laparoscopic and open surgery. Am J Surg 2001;182:720.

9. Kercher KW, Joels CS, Matthews BD, Lincourt AE, Smith TI, Heniford BT. Hand-assisted surgery improves outcomes for laparoscopic nephrectomy. Am Surg 2003;69:1061.

10. Wolf JS Jr, Moon TD, Nakada SY. Hand assisted laparoscopic nephrectomy: Comparison to standard laparoscopic nephrectomy. J Urol 1999;162:806.

11. Hanna GB, Elamass M, Cuschieri A. Ergonomics of hand-assisted laparoscopic surgery. Semin Laparosc Surg 2001;8:92.
12. Berguer R, Rab GT, Abu-Ghaida H, Alarcon A, Chung J. A comparison of surgeons' posture during laparoscopic and open surgical procedures. Surg Endosc 1997;11:139.

13. Premoli J, Durfee W, Skemp N, Monga M. Forearm compression by laparoscopic hand-assist devices [abstract]. J Endourol 2002; 16(suppl): 18 .

14. Berguere R, Forkey DL, Smith WD. Ergonomic problems associated with laparoscopic surgery. Surg Endosc 1999;13:466.

15. Quick NE, Gillette JC, Shapiro GL, Adrales GL, Gerlach D, Park AE. The effect of using laparoscopic instruments on muscle activation patterns during minimally invasive surgical training procedures. Surg Endosc 2003;17:462.

16. Berguer R, Forkey DL, Smith WD. The effect of laparoscopic instrument working angle on surgeons' upper extremity workload. Surg Endosc 2001;15:1027.

17. Berquer R, Smith WD, Davis S. An ergonomic study of the optimum operating table height for laparoscopic surgery. Surg Endosc 2002;16:416.

18. van Veelen MA, Kazemier G, Koopman J, Goossens RH, Meijer DW. Assessment of the ergonomically optimal operating surface height for laparoscopic surgery. J Laparoendosc Adv Surg Tech A 2002;12:47.

19. Uhrich ML, Underwood RA, Standeven JW, Soper NJ, Engsberg JR. Assessment of fatigue, monitor placement, and surgical experience during simulated laparoscopic surgery. Surg Endosc 2002;16:635.

20. Hanna GB, Shimi SM, Cuschieri A. Task performance in endoscopic surgery is influenced by location of the image display. Ann

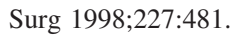

Address reprint requests to: J. Stuart Wolf, Jr., M.D. Dept. of Urology and Minimally Invasive Urology University of Michigan 1500 E. Medical Center Drive Ann Arbor, MI 48109-0330

E-mail:wolfs@umich.edu 


\section{This article has been cited by:}

1. Dimitrios Stefanidis, Fikre Wang, James R. Korndorffer, J. Bruce Dunne, Daniel J. Scott. 2009. Robotic assistance improves intracorporeal suturing performance and safety in the operating room while decreasing operator workload. Surgical Endoscopy . [CrossRef]

2. R. H. Schatte Olivier, C. D. P. Hullenaar, J. P. Ruurda, I. A. M. J. Broeders. 2009. Ergonomics, user comfort, and performance in standard and robot-assisted laparoscopic surgery. Surgical Endoscopy 23:6, 1365-1371. [CrossRef]

3. Aditya Bagrodia , Jay D. Raman . 2009. Ergonomics Considerations of Radical Prostatectomy: Physician Perspective of Open, Laparoscopic, and Robot-Assisted TechniquesErgonomics Considerations of Radical Prostatectomy: Physician Perspective of Open, Laparoscopic, and Robot-Assisted Techniques. Journal of Endourology 23:4, 627-633. [Abstract] [PDF] [PDF Plus]

4. J Stuart Wolf Jr. 2005. Devices for hand-assisted laparoscopic surgery. Expert Review of Medical Devices 2:6, 725-730. [CrossRef]

5. 2005. LiteratureWatchLiteratureWatch. Journal of Endourology 19:8, 1045-1062. [Citation] [PDF] [PDF Plus] 